\title{
Multiplicity dependence of $D$ and $J / \psi$ production in small collision systems
}

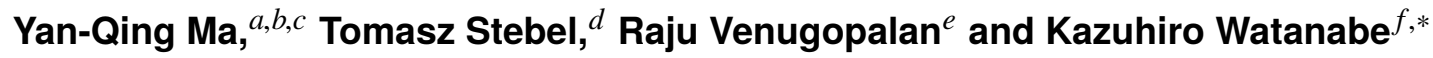 \\ ${ }^{a}$ School of Physics and State Key Laboratory of Nuclear Physics and Technology, Peking University, \\ Beijing 100871, China. \\ ${ }^{b}$ Center for High Energy Physics, Peking University, Beijing 100871, China. \\ ${ }^{c}$ Collaborative Innovation Center of Quantum Matter, Beijing 100871, China. \\ ${ }^{d}$ Institute of Theoretical Physics, Jagiellonian University, S.Łojasiewicza 11, 30-348 Kraków, Poland. \\ ${ }^{e}$ Physics Department, Brookhaven National Laboratory, Upton, New York 11973-5000, USA. \\ ${ }^{f}$ Theory Center, Jefferson Laboratory, Newport News, Virginia 23606, USA. \\ E-mail: yqma@pku.edu.cn, tomasz.stebel@uj.edu.pl, raju@bnl.gov, \\ watanabe@jlab.org
}

\begin{abstract}
We discuss open heavy flavor (D-meson) and quarkonium $(J / \psi)$ production in high multiplicity proton-proton $(p+p)$ and proton-nucleus $(p+A)$ collisions at the LHC in the impact parameter dependent saturation model. A key ingredient in our calculations is the fluctuations of the gluon saturation scale at large- $x$ at each impact parameter, reflecting rare parton configurations inside the projectile and target. Such initial state fluctuations have been used to describe the multiplicity distribution of $D$-meson and $J / \psi$ production yield at mid rapidity in small collision systems at the LHC. In these proceedings, we examine the rapidity dependence of $J / \psi$ production and emphasize its importance in constraining initial state models of high multiplicity events in small systems.
\end{abstract}

HardProbes 2020

1-6 June 2020

Austin, Texas

${ }^{*}$ Speaker 


\section{Introduction}

The underlying mechanism of particle production in high multiplicity events in the small systems formed in proton-proton $(p+p)$ and proton-nucleus $(p+A)$ collisions has attracted much attention because of their promising role in isolating initial state physics from that of final state effects. Heavy-flavor and quarkonium production in high multiplicity $p+p$ and $p+A$ collisions are useful observables to probe QCD dynamics at high event activity. A crucial issue to be resolved is whether initial state effects can provide a systematic description of light hadron, open heavy flavor, and quarkonium production in high multiplicity events.

The Color Glass Condensate (CGC) effective field theory (EFT) [1] is a useful tool that allows us to explore the initial state physics of gluon saturation that provides a mechanism to generate high multiplicity phenomena. Such high multiplicity events corresponding to multiplicities $N_{c h} \gg\left\langle N_{c h}\right\rangle$, the minimum bias multiplicity, however, probe the dynamics of saturation in rare parton configurations inside hadrons and nuclei [2]; the theoretical framework for such rare events in the CGC EFT requires further development. They can nevertheless be modeled by allowing the values of the saturation scales at large $x \sim 0.01$ to be larger than those in minimum bias events, and evolving the corresponding distributions to smaller values of $x$ employing the CGC renormalization group framework. In previous studies [3, 4], we found that this simple modification of large $x$ initial conditions provides a good description of some features of the data on $D$-meson and $J / \psi$ production in high multiplicity $p+p$ and $p+A$ collisions at the LHC. In this writeup, we will outline some of the principal results of our work and briefly discuss how the rapidity dependence of the LHC data can further help constrain initial state models of rare events.

\section{Framework}

Analytical results for particle multiplicities can be obtained in a $k_{t}$-factorized "dilute-dense" approximation of the CGC EFT. In this approximation, the yield of an inclusive gluon mini jet in a dilute-dense system at an impact parameter $b_{\perp}$ is given by [5]

$$
\frac{d N_{g}\left(\boldsymbol{b}_{\perp}\right)}{d^{2} \boldsymbol{q}_{\perp} d y}=\frac{\alpha_{s}}{(2 \pi)^{3} \pi^{3} C_{F}} \frac{1}{q_{\perp}^{2}} \int_{\boldsymbol{k}_{\perp}} \int_{\boldsymbol{s}_{\perp}} \frac{d \phi_{x_{1}}^{g, g}\left(\boldsymbol{k}_{\perp} \mid \boldsymbol{s}_{\perp}\right)}{d^{2} \boldsymbol{s}_{\perp}} \frac{d \phi_{x_{2}}^{g, g}\left(\boldsymbol{q}_{\perp}-\boldsymbol{k}_{\perp} \mid \boldsymbol{s}_{\perp}-\boldsymbol{b}_{\perp}\right)}{d^{2} \boldsymbol{s}_{\perp}}
$$

where $\int_{\boldsymbol{k}_{\perp}} \equiv \int d^{2} \boldsymbol{k}_{\perp}$ and $x_{1,2}$ denote longitudinal momentum fractions of an incoming gluon inside the dilute projectile and the dense target, respectively. The impact parameter dependent two point function (gluon distribution function) reads

$$
\frac{d \phi_{x}^{g, g}\left(\boldsymbol{k}_{\perp} \mid \boldsymbol{s}_{\perp}\right)}{d^{2} \boldsymbol{s}_{\perp}}=\frac{k_{\perp}^{2} N_{c}}{4 \alpha_{s}} \int d^{2} \boldsymbol{r}_{\perp} e^{i \boldsymbol{r}_{\perp} \cdot \boldsymbol{k}_{\perp}}\left[1-\mathcal{N}_{x}\left(\boldsymbol{r}_{\perp}, \boldsymbol{s}_{\perp}\right)\right]^{2}
$$

where $\mathcal{N}_{x}$ denotes the dipole amplitude in the fundamental representation. The transverse coordinate $\boldsymbol{s}_{\perp}$ runs over the transverse plane of the projectile or target. The impact parameter $\boldsymbol{b}_{\perp}$ will be integrated over eventually in our calculations: $d N_{c h}=\int d^{2} \boldsymbol{b}_{\perp} d N_{c h}\left(\boldsymbol{b}_{\perp}\right) / \int d^{2} \boldsymbol{b}_{\perp}$. In our calculations, we take $b_{\perp}^{\max }=1[\mathrm{fm}]$ for the proton and $b_{\perp}^{\max }=R_{A}=1.12 A^{1 / 3}-0.86 A^{-1 / 3}[\mathrm{fm}]$ for a nucleus with $A$ atomic mass. Similarly, the $k_{t}$-factorized formula for a heavy quark pair $(c \bar{c})$ 
production is given by $[6,7]$

$$
\frac{d N_{c \bar{c}}\left(\boldsymbol{b}_{\perp}\right)}{d^{2} \boldsymbol{p}_{c \perp} d^{2} \boldsymbol{q}_{\bar{c} \perp} d y_{c} d y_{\bar{c}}}=\frac{\alpha_{s}^{2}}{(2 \pi)^{10} C_{F}} \int_{\boldsymbol{k}_{\perp \perp}, \boldsymbol{k}_{\perp}} \frac{\Xi}{k_{1 \perp}^{2} k_{2 \perp}^{2}} \int_{s_{\perp}} \frac{d \phi_{x_{1}}^{g, g}\left(\boldsymbol{k}_{1 \perp} \mid \boldsymbol{s}_{\perp}\right)}{d^{2} \boldsymbol{s}_{\perp}} \frac{d \phi_{x_{2}}^{q \bar{q}, g}\left(\boldsymbol{k}_{2 \perp}, \boldsymbol{k}_{\perp} \mid \boldsymbol{s}_{\perp}-\boldsymbol{b}_{\perp}\right)}{d^{2} \boldsymbol{s}_{\perp}},
$$

where $\Xi$ denotes a hard scattering contribution, and we have introduced the impact parameter dependent multi-point function for the target,

$$
\frac{d \phi_{x}^{q \bar{q}, g}\left(\boldsymbol{k}_{2 \perp}, \boldsymbol{k}_{\perp} \mid \boldsymbol{s}_{\perp}\right)}{d^{2} \boldsymbol{s}_{\perp}}=\frac{k_{2 \perp}^{2} N_{c}}{4 \alpha_{s}} \int d^{2} \boldsymbol{r}_{\perp} d^{2} \boldsymbol{r}_{\perp}^{\prime} e^{i \boldsymbol{r}_{\perp} \cdot \boldsymbol{k}_{\perp}} e^{i \boldsymbol{r}_{\perp}^{\prime} \cdot\left(\boldsymbol{k}_{2 \perp}-\boldsymbol{k}_{\perp}\right)} \mathcal{N}_{x}\left(\boldsymbol{r}_{\perp}, \boldsymbol{s}_{\perp}\right) \mathcal{N}_{x}\left(\boldsymbol{r}_{\perp}^{\prime}, \boldsymbol{s}_{\perp}\right) .
$$

In the IP-Sat model, the dipole amplitude reads $\mathcal{N}_{x}\left(\boldsymbol{r}_{\perp}, \boldsymbol{s}_{\perp}\right)=1-\exp \left[-\frac{\pi^{2}}{2 N_{c}} r_{\perp}^{2} \alpha_{s}\left(\mu^{2}\right) x f_{g}\left(x, \mu^{2}\right) T_{p}\left(\boldsymbol{s}_{\perp}\right)\right]$ where $x f_{g}$ is the collinear gluon distribution function, and $T_{p}$ denotes a transverse profile function of the gluon's density inside the proton. The IP-Sat model has been compared to combined HERA data in Ref. [8]. In $p+A$ collisions, the dipole amplitude for the target nucleus can be approximated to a coherent scattering form [9]: $\mathcal{N}_{x}^{A}\left(\boldsymbol{r}_{\perp}, \boldsymbol{s}_{\perp}\right) \approx 1-\exp \left[-A \hat{T}_{A}\left(\boldsymbol{s}_{\perp}\right) \int d^{2} \boldsymbol{s}_{\perp}^{\prime} \mathcal{N}_{x}\left(\boldsymbol{r}_{\perp}, \boldsymbol{s}_{\perp}^{\prime}\right)\right]$ with $\hat{T}_{A}$ a nuclear thickness function.

We will employ the Local-Parton-Hadron-Duality (LHPD) principle to describe the hadronization of the gluon jet [10]; The transverse momentum of a hadron $h$ is given by $p_{\perp}^{h}=\langle z\rangle q_{\perp}$ with $p_{\perp}^{h}=\left|\boldsymbol{p}_{\perp}^{h}\right|$ and $q_{\perp}=\left|\boldsymbol{q}_{\perp}\right| .\langle z\rangle$ denotes the average longitudinal momentum fraction of the gluon fragmenting into the hadron $h$, but the multiplicity distribution does not depend on $\langle z\rangle$ : $d N_{c h} / d \eta \sim d N_{g} / d \eta$. The differential yield for $D$ meson production is given by taking a convolution of the $c \bar{c}$ yield with a heavy quark fragmentation function as implemented in Ref. [3]. We use KKKS08 set [11] to compute the $D$-meson yield. For $J / \psi$ production, we will simply use the CGC+Improved Color Evaporation Model (ICEM) framework [12], in which the hadronization of ${ }^{3} S_{1}^{[8]}$ intermediate state of the $c \bar{c}$ pair dominates over other intermediate states.

\section{Numerical Results}

Figure 1 shows the mean transverse momentum of a produced hadron $h$ as a function of $N_{c h}$ at the LHC. The larger the initial saturation scales, the larger $N_{c h}$ becomes. To reach higher $N_{c h}$ at mid rapidity, we need to increase the saturation scales for both the projectile and target at $x=0.01: Q_{s}^{2}=N Q_{0}^{2}$ with $N=0.5,1,2, \cdots$ and $Q_{0}^{2}$ being the saturation scale in MB events. We found that $\left\langle p_{\perp}^{h}\right\rangle=\left\langle\langle z\rangle q_{\perp}\right\rangle$ underestimates the LHC data at high event activity (see dashed curves). At large event activity, the semihard rescatterings before the gluon jet hadronizes enable the gluon jet to gain larger transverse momenta, as captured by a simple random walk formula $\left\langle p_{\perp}^{h}\right\rangle=\sqrt{\left\langle\langle z\rangle q_{\perp}\right\rangle^{2}[1+c(n-1)]}$ with $n=N_{c h} /\left\langle N_{c h}\right\rangle$. Tuning $c$, we can describe the LHC data (see solid curves). In Ref. [10], a different b-CGC model for the dipole amplitude and a constant intrinsic transverse momentum of the gluon jet was used to describe the data; we are unable to reproduce the results of this paper. Note that for quarkonia, unlike lighter hadrons, the effect of semihard rescatterings is unlikely to influence the behavior of $\left\langle p_{\perp}\right\rangle$ in high multiplicity events. See the discussion in Ref. [3].

Figure 2 (Left) shows the $N_{c h}$ distribution of $D$-meson yield at mid rapidity at the LHC. The IP-Sat model with KKKS08 set can describe the LHC data in both $p+p$ and $p+A$ collisions. For 


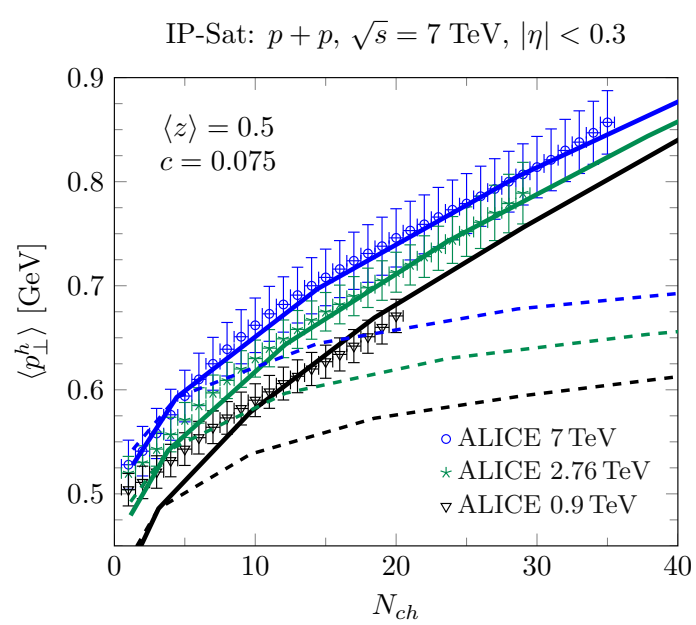

Figure 1: Mean transverse momentum of produced light hadron vs. $N_{c h}$ in $p+p$ collisions at the LHC. The energy dependence shows in different colors. Solid (dashed) curves correspond to results with (without) the semihard rescattering effect. See text.
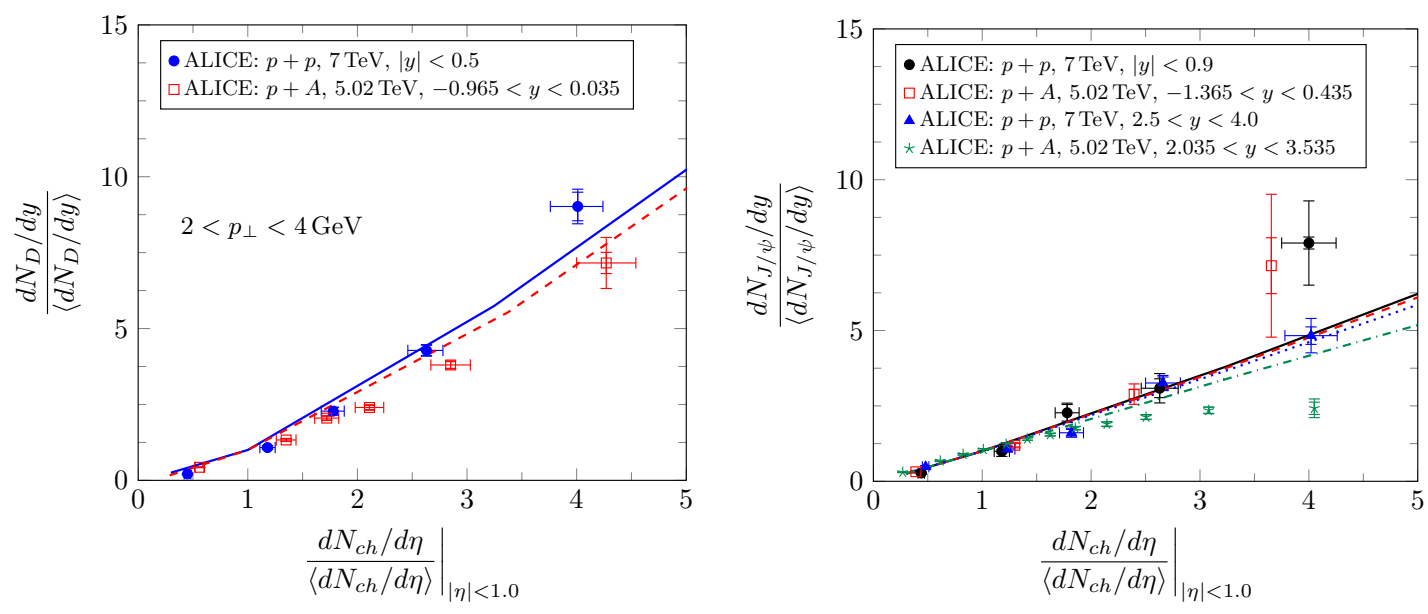

Figure 2: (Left): $N_{c h}$ dependence of relative yield of $D$ meson (average $D_{0}, D^{*+}, D^{+}$) at mid rapidity in $p+p$ (blue solid) and $p+A$ (red dashed) collisions. Data from [13, 14]. (Right): Results for $J / \psi$ production at forward rapidity in $p+p(p+A)$ collisions at $\sqrt{s}=7 \mathrm{TeV}(5.02 \mathrm{TeV})$ in the CGC+ICEM model. Black solid: $p+p$ mid, Red dashed: $p+A$ mid, Blue dotted: $p+p$ forward, Green dash-dotted: $p+A$ forward. Data are taken from Refs. $[15,16]$.

$J / \psi$ production at mid rapidity in small systems, the IP-Sat+ICEM model captures the correct trend in the growth of the $J / \psi$ yield at large event activity. See the black solid and red dashed curves in Fig. 2 (Right).

With the same parametrization, we computed $J / \psi$ production at forward rapidity. As shown by the blue dotted and green dash-dotted curves, the $N_{c h}$ distribution of the $J / \psi$ yield in $p+p$ collisions is similar to the one in $p+A$ collisions up to $d N_{c h} /\left\langle d N_{c h}\right\rangle \sim 4$, regardless of the rapidity. However, the LHC data indicate that the $J / \psi$ yield at forward rapidity is suppressed compared to 
the yield at mid rapidity in $p+A$ collisions for $d N_{c h} /\left\langle d N_{c h}\right\rangle>2$; while our model shows a small suppression, it does not capture that seen in the data. Whether this additional suppression can be simply accommodated or requires a significant change in the model is under active investigation.

\section{Acknowledgments}

Support of the Polish National Science Center Grant No. 2019/32/C/ST2/00202 is kindly acknowledged. R.V. is supported by the U.S. Department of Energy under Contract No. DESC0012704. His work is also supported by the US DOE within the framework of the TMD Theory Topical Collaboration. K.W. is supported by Jefferson Science Associates, LLC under U.S. DOE Contract No. DE-AC05-06OR23177.

\section{References}

[1] F. Gelis, E. Iancu, J. Jalilian-Marian and R. Venugopalan, Ann. Rev. Nucl. Part. Sci. 60, 463-489 (2010).

[2] K. Dusling and R. Venugopalan, Phys. Rev. D 87, no.9, 094034 (2013).

[3] Y. Q. Ma, P. Tribedy, R. Venugopalan and K. Watanabe, Phys. Rev. D 98, no.7, 074025 (2018).

[4] Y. Q. Ma, P. Tribedy, R. Venugopalan and K. Watanabe, Nucl. Phys. A 982, 747-750 (2019).

[5] J. P. Blaizot, F. Gelis and R. Venugopalan, Nucl. Phys. A 743, 13-56 (2004).

[6] F. Gelis and R. Venugopalan, Phys. Rev. D 69, 014019 (2004).

[7] J. P. Blaizot, F. Gelis and R. Venugopalan, Nucl. Phys. A 743, 57-91 (2004).

[8] A. H. Rezaeian, M. Siddikov, M. Van de Klundert and R. Venugopalan, Phys. Rev. D 87, no.3, 034002 (2013).

[9] H. Kowalski, T. Lappi and R. Venugopalan, Phys. Rev. Lett. 100, 022303 (2008).

[10] A. H. Rezaeian, Phys. Lett. B 727, 218-225 (2013).

[11] T. Kneesch, B. A. Kniehl, G. Kramer and I. Schienbein, Nucl. Phys. B 799, 34-59 (2008).

[12] Y. Q. Ma, R. Venugopalan, K. Watanabe and H. F. Zhang, Phys. Rev. C 97, no.1, 014909 (2018).

[13] J. Adam et al. [ALICE], JHEP 09, 148 (2015).

[14] J. Adam et al. [ALICE], JHEP 08, 078 (2016).

[15] B. Abelev et al. [ALICE], Phys. Lett. B 712, 165-175 (2012).

[16] D. Adamová et al. [ALICE], Phys. Lett. B 776, 91-104 (2018). 